\title{
Effect of transcription factor ZBTB20 on mouse pituitary development
}

\author{
Q. Dong ${ }^{1,2}$, X.Y. Chen ${ }^{2}$ and G.M. Li ${ }^{1}$ \\ 'Department of Pediatrics, Shandong Provincial Hospital, \\ Affiliated to Shandong University, Ji'nan, Shandong Province, China \\ 2Department of Pediatrics, Central Hospital of Tai'an, Tai'an, Shandong, China \\ Corresponding author: G.M. Li \\ E-mail: maikjicnes@163.com
}

Genet. Mol. Res. 14 (4): 17622-17629 (2015)

Received August 10, 2015

Accepted October 18, 2015

Published December 21, 2015

DOI http://dx.doi.org/10.4238/2015.December.21.35

ABSTRACT. Pituitary, a critical component in the neuroendocrine system, plays an indispensable role in the regulation of body growth. The transcriptional factor ZBTB20 is widely expressed in brain tissues and participates in hippocampal development; however, the detailed molecular mechanism remains unknown. Therefore, the aim of this study was to investigate the effect of ZBTB20 on mouse pituitary development and related mechanisms in ZBTB20 gene knockout mice. The expressional profiles of ZBTB20 in various neuroendocrinal cells during the different developmental stages (from E10 to P0) were described by immunofluorescence staining. A ZBTB20 gene knockout mouse model was then generated. Real-time polymerase chain reaction and western blotting assays were used to detect the levels of five hormones: growth hormone $(\mathrm{GH})$, prolactin (PRL), luteinizing hormone ( $\mathrm{LH})$, follicle-stimulating hormone (FSH), and thyroidstimulating hormone (TSH). ZBTB20 protein expression was identified from E14 until birth. A majority of the pituitary endocrinal cells were ZBTB20positive. In ZBTB20 knockout mice, the level of GH decreased by half and $\mathrm{PRL}$ expression was eliminated. No significant change was observed in the other three hormones ( $\mathrm{LH}, \mathrm{FSH}$, and TSH). ZBTB20, an important transcriptional factor in pituitary development, is mainly responsible for the 
terminal differentiation of prolactin-secreting cells, thereby regulating the secretion of the pituitary hormones.

Key words: ZBTB20 transcriptional factor; Gene knockout mouse; Pituitary development; Developmental regulation

\section{INTRODUCTION}

The pituitary, an indispensable component of the neuroendocrinological system, maintains the body development and homeostasis by facilitating communications between the hypothalamus and peripheral target organs, thereby playing an important role in body development and metabolism. The normal development and function of pituitary is dependent on various signaling pathways and transcriptional factors, all of which may interfere with development and interrupt endocrinal homeostasis (Franceschi et al., 2011; Baranowski and Hogler, 2012). Recent progress in genetic manipulation of animals has helped us gain more knowledge regarding the role of mutations and transcriptional factors in pituitary development; however, the molecular mechanism of this process remains unknown (Nanbu-Wakao et al., 2000). Current research is focused on two major pathways that have been postulated to affect pituitary development: the proliferation and differentiation of stem cells in the pituitary, and the proliferation of differentiated cells. Different endocrinal cell groups have been reported to regulate cellular proliferation and development during different stages, with the most important regulatory functions being derived from pituitary hormones (Wu et al., 2003; Cabrera et al., 2013). Scholars have gained some knowledge about the regulation of pituitary development; however, the exact mechanism of this process remains unclear. As even a single change in known signaling molecules and transcriptional factors can induce various phenotype and hormone level variations, a greater number of pituitary development-related genes must be investigated further (Reynaud et al., 2011). Previous in vivo studies have demonstrated that the ZBTB20 transcriptional factor, expressed in the pituitary gland, plays a critical role in mouse development (Shillingford et al., 2003). In this study, the physiological role of the ZBTB20 transcriptional factor in pituitary development was further investigated in an attempt to gain further knowledge of the molecular mechanisms underlying pituitary growth, and to provide a theoretical basis for further studies regarding hypopituitarism.

\section{MATERIAL AND METHODS}

\section{Animals}

ZBTB20 gene knockout mice were generated in-house using C57/BL6 mice. Normal C57/ BL6 mice were used as controls. The experimental protocol was pre-approved by the Animal Ethics Committee of Shandong Provincial Hospital.

\section{Immunofluorescence assays}

Mouse pituitary tissues were extracted, fixed, and embedded in paraffin. The embedded tissues were sectioned, dewaxed, and subjected to antigen retrieval using hot citric acid buffer or EDTA buffer (according to the requirements of different cytoplasmic/nuclear antigens). Endogenous 
peroxidase activity was quenched by $3 \%$ hydrogen peroxide (v/v). Non-specific binding was blocked by $50 \mu \mathrm{L}$ fetal bovine serum for $1 \mathrm{~h}$. The sections were incubated overnight with primary antibodies (Santa Cruz Biotechnology, Santa Cruz, CA, USA); subsequently, the sections were incubated with a biotin-labeled secondary antibody (Beyotime Biotechnology Inc., Beijing, China). The signals were developed with horseradish peroxidase (Boster Biologics, Pleasanton, CA, USA). The sections were incubated with a fluorescent dye, counterstained with DAPI in the dark, and the images were captured under an inverted fluorescent microscope (Olympus, Tokyo, Japan).

A total of five fields were randomly selected from each of the five slices. The staining score was described as (1) positive rate score $+(2)$ intensity score. The positive rate score was the category of positive cells (\%) in a field, filed as: $0,<5 \% ; 1,6-25 \% ; 2,26-50 \% ; 3,51-75 \%$; and 4 , $>75 \%$. The intensity score was as follows: 0 , no color; 1 , light yellow; 2 , yellow-brown; and 3 , dark brown. The final staining score was categorized as: negative (-), 0; weak positive (+), 1-2; positive $(++), 3-4$; and strong positive (+++), 5-7.

\section{Double-immunofluorescence labeling}

Pituitary tissue sections were processed as described in the previous section. The slides were subjected to peroxidase quenching, gently washed with PBS, and subsequently incubated overnight with dual-primary antibodies. Two types of secondary antibodies with CY3 or Hoechst 33258 dyes were sequentially added for biotin incubation. The images were captured under a fluorescence microscope.

\section{Real-time reverse transcriptase-polymerase chain reaction (PCR)}

Pituitary tissues $(0.1 \mathrm{~g})$ were added to $0.1 \mathrm{~mL}$ TRIzol (Invitrogen, Carlsbad, CA, USA) for total RNA extraction, according to the instructions of the manufacturer. The extracted RNA $(2 \mu \mathrm{g})$ was used as the template for the synthesis of cDNA by in vitro reverse transcription. The PCR mixture was composed of $2 \mu \mathrm{L}$ cDNA and the Platinum SYBR Green qPCR SuperMix (Invitrogen). The reaction conditions were set as follows: pre-denaturation at $95^{\circ} \mathrm{C}$ for $5 \mathrm{~min}$, followed by 30 cycles of denaturation at $94^{\circ} \mathrm{C}$ for $30 \mathrm{~s}$, annealing at $60^{\circ} \mathrm{C}$ for $30 \mathrm{~s}$, and extension at $72^{\circ} \mathrm{C}$ for 60 s. The PCR products were separated by agarose gel electrophoresis using a $1 \%$ gel. The images were normalized against the 18s rRNA, which was used as an internal reference control. Semiquantitative analysis was performed using integrated optical density for the calculation of relative expression levels. Sequences of primers used are listed in Table 1.

\begin{tabular}{|c|c|c|}
\hline & Forward primer & Reverse primer \\
\hline $\mathrm{GH}$ & 5'-AAGGTGAAGAGCATCATAACCCT-3' & 5'-TCACGCCTTTCATAACACATTCC-3' \\
\hline PRL & 5'-GGAGGTGGTGATAGCCGGTAT-3' & 5'-TGGGTAATCCATAGAGCCCAG-3' \\
\hline LH & 5'-CCAGCCTGAGGGCTTACTG-3' & 5'-СTCCATTGACTGTGACATCTCG-3' \\
\hline $\mathrm{FSH}$ & 5'-CTGGACCAGAGACCCTTTGC-3' & 5'-GGACGGGGACTTCTGAGTCTT-3' \\
\hline TSH & 5'-GAGACCCCTGTGTCGGTTC-3' & 5'-CTGCGTGTGTGAAATGTCATTG-3' \\
\hline ACTH & 5'-AGAGCCCCATCTGTCCTCTC-3' & 5'-ACTGGTAGTCTGCAAAACCAAA-3' \\
\hline ZBTB20 & 5'-GACC ACTCGC ATTCCTTT-3' & 5'-GGC ATTGTGAG AC ATCCC-3' \\
\hline 18s RNA & 5'-TCAGCGTG AAGATGGCTTCG-3' & 5'-TCAGATCTGGAAGGGGAAGGAA-3' \\
\hline
\end{tabular}

$\mathrm{GH}=$ growth hormone; $\mathrm{PRL}=$ prolactin; $\mathrm{LH}=$ luteinizing hormone; $\mathrm{FSH}=$ follicle-stimulating hormone; $\mathrm{TSH}=$ thyroidstimulating hormone. 


\section{Western blot}

Total proteins were extracted from the pituitary using a lysis buffer, and separated via sodium dodecyl sulfate polyacrylamide gel electrophoresis (SDS-PAGE). The separated proteins were transferred to a polyvinylidene difluoride (PVDF) membrane using a semi-dry method (BioRad, Hercules, CA, USA). The membrane was then incubated overnight with primary antibodies against growth hormone $(\mathrm{GH})$, prolactin ( $\mathrm{PRL}$ ), luteinizing hormone $(\mathrm{LH})$, follicle-stimulating hormone (FSH), thyroid-stimulating hormone (TSH), and ZBTB20 (1:500; Santa Cruz Biotechnology). The membrane was then incubated with a horseradish peroxidase-conjugated goat anti-rabbit secondary antibody (1:1000; Beyotime Biotechnology) for $1 \mathrm{~h}$. The membrane was then developed by electrochemiluminescence (ECL). The optical density was analyzed using an imaging software (ImageQuant TL, GE Healthcare, Pittsburgh, PA, USA).

\section{Statistical analysis}

All data were analyzed using the SPSS 21.0 software package (IBM, Armonk, NY, USA). Data fitted with normal distribution are reported as means \pm standard deviation (SD). Data that did not fit normal distribution are reported as the median and quartile values. Between-group comparisons of parametric data were performed using the LSD test, while the non-parametric data were compared by Kruskal-Wallis H-test (for multiple groups) or Mann-Whitney U-test (for two groups). A statistical significance was defined when $\mathrm{P}<0.05$.

\section{RESULTS}

\section{Expressional profiles of ZBTB20 in pituitary at different stages}

Immunofluorescence was used to describe the expression of ZBTB20 proteins during the different developmental stages (from E10 to P0). The ZBTB20 protein was initially identified in E14 pituitary. The number of ZBTB20-positive cells increased significantly from E15 and E16 until birth. Both anterior and posterior pituitary lobes contained large amounts of ZBTB20 (Figure 1 and Table 2).

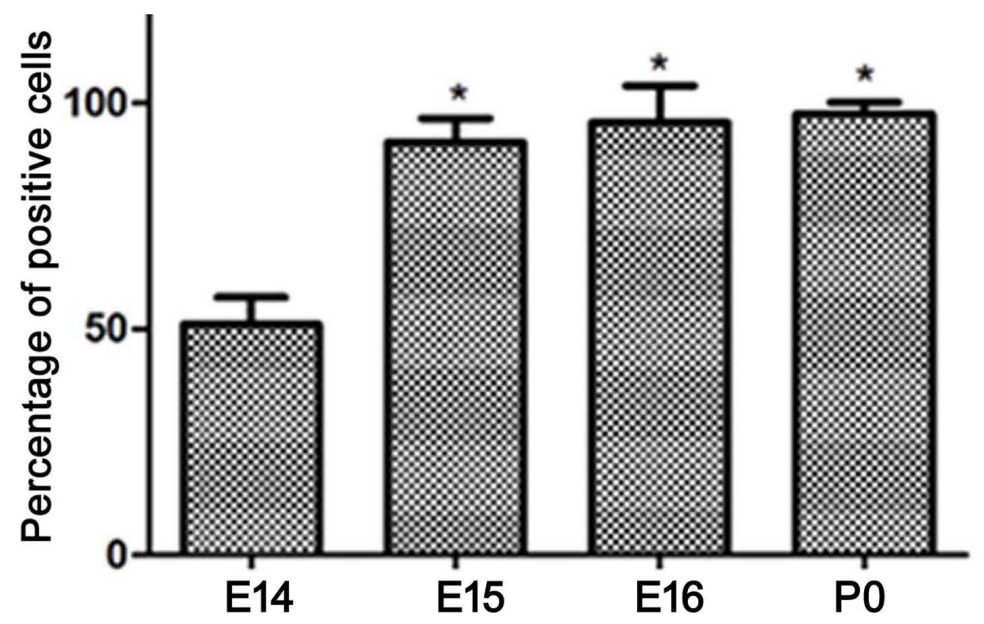

Figure 1. Expression of ZBTB20 in mouse pituitary. ${ }^{*} P<0.05$ compared to $E 14$. 
Table 2. ZBTB20 expression profiles.

\begin{tabular}{|c|c|c|c|c|c|c|}
\hline Time point & $\mathrm{N}$ & - & + & ++ & +++ & Positive cell (\%) \\
\hline E14 & 31 & 15 & 16 & 0 & 0 & 51.61 \\
\hline E15 & 46 & 4 & 15 & 17 & 10 & 91.30 \\
\hline E16 & 47 & 2 & 16 & 21 & 8 & 95.74 \\
\hline P0 & 43 & 1 & 18 & 15 & 9 & 97.67 \\
\hline
\end{tabular}

Weak positive (+); positive (++); strong positive (+++) cells.

\section{Distribution of ZBTB20 in different pituitary endocrine cells}

We further tested the expression of ZBTB20 in different pituitary endocrinal cells (eosinophilic, basophilic, and chromophobe cells). Immunofluorescence staining (Figure 2 and Table 3) revealed the existence of ZBTB20 in most endocrine cells, as supported by the co-localization of ZBTB20 and different hormones, including GH, PRL, LH, FSH, TSH, and adrenocorticotrophic hormone $(\mathrm{ACTH})$.

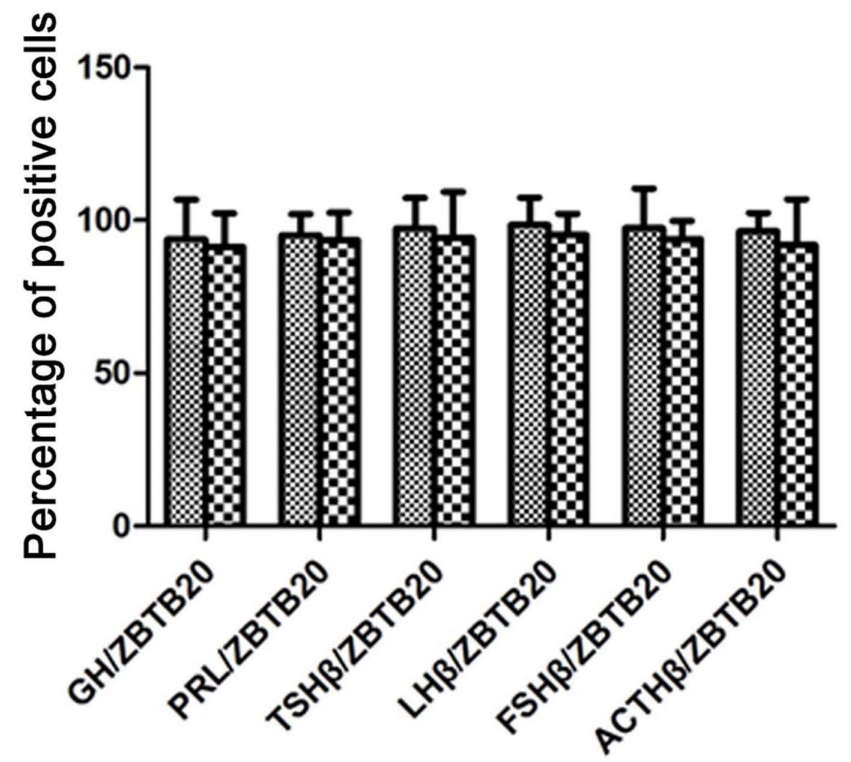

Figure 2. ZBTB20 expression in different endocrinal cells of the pituitary.

Table 3. ZBTB20 expression profiles across various endocrinal cell groups.

\begin{tabular}{lccccc}
\hline Hormones & - & + & ++ & +++ & Percentage \\
\hline GH/ZBTB20 & $4 / 6$ & $21 / 26$ & $24 / 19$ & $15 / 18$ & $93.75 / 91.30$ \\
PRL/ZBTB20 & $3 / 4$ & $26 / 23$ & $17 / 18$ & $15 / 17$ & $95.08 / 93.55$ \\
TSHB/ZBTB20 & $2 / 5$ & $24 / 29$ & $21 / 32$ & $28 / 22$ & $97.33 / 94.32$ \\
LHB/ZBTB20 & $1 / 4$ & $18 / 24$ & $15 / 19$ & $29 / 36$ & $98.41 / 95.18$ \\
FSHß/ZBTB20 & $2 / 6$ & $27 / 33$ & $24 / 27$ & $24 / 30$ & $97.40 / 93.75$ \\
ACTHB/ZBTB20 & $3 / 7$ & $24 / 26$ & $27 / 26$ & $30 / 28$ & $96.43 / 91.95$ \\
\hline
\end{tabular}

$\overline{\mathrm{GH}}=$ growth hormone; $\mathrm{PRL}$ = prolactin; $\mathrm{LH}=$ luteinizing hormone; $\mathrm{FSH}$ = follicle-stimulating hormone; $\mathrm{TSH}=$ thyroidstimulating hormone. 


\section{Real-time PCR}

Real-time PCR was performed in ZBTB20 gene knockout (KO) mice to measure the mRNA levels of different hormones in pituitary cells. As shown in Table 4, hardly any PRL mRNA was detected in $\mathrm{KO}$ mice, and $\mathrm{GH}$ mRNA expression was only about half of that seen in control animals. However, the mRNA level of the other three hormones, did not differ significantly in the pituitary.

\begin{tabular}{|c|c|c|c|c|}
\hline Target gene & Wild type & ZBTB20 KO & $t$ value & $P$ value \\
\hline ZBTB20 & $1.01 \pm 0.31$ & 0.00 & 5.634 & 0.000 \\
\hline PRL & $1.02 \pm 0.56$ & 0.00 & 5.821 & 0.000 \\
\hline $\mathrm{GH}$ & $1.70 \pm 0.56$ & $0.86 \pm 0.45$ & 3.647 & 0.002 \\
\hline АСТН $\beta$ & $1.16 \pm 0.69$ & $1.22 \pm 0.36$ & 1.123 & 0.734 \\
\hline LH $\beta$ & $1.02 \pm 0.23$ & $1.20 \pm 0.31$ & 1.254 & 0.621 \\
\hline $\mathrm{FSH} \beta$ & $1.01 \pm 0.22$ & $1.21 \pm 0.21$ & 1.325 & 0.601 \\
\hline
\end{tabular}

$\mathrm{GH}=$ growth hormone; $\mathrm{PRL}=$ prolactin; $\mathrm{LH}=$ luteinizing hormone; $\mathrm{FSH}=$ follicle-stimulating hormone; $\mathrm{TSH}=$ thyroidstimulating hormone.

\section{Protein levels of released hormones}

The protein levels of all five pituitary-derived hormones were also evaluated by western blot. The results obtained were consistent with those of mRNA (Table 5).

Table 5. Protein levels of pituitary-releasing hormones.
\begin{tabular}{lcccc} 
\\
Hormones & Wild type & ZBTB20 KO & t value & P value \\
\hline ZBTB20 & $1.02 \pm 0.21$ & 0.00 & 5.867 & 0.000 \\
PRL & $1.03 \pm 0.06$ & 0.00 & 5.689 & 0.000 \\
GH & $1.02 \pm 0.05$ & $0.51 \pm 0.05$ & 3.543 & 0.002 \\
ACTH $\beta$ & $1.06 \pm 0.02$ & $1.32 \pm 0.25$ & 1.321 & 0.667 \\
LH $\beta$ & $1.04 \pm 0.36$ & $1.31 \pm 0.21$ & 1.226 & 0.620 \\
FSH $\beta$ & $1.01 \pm 0.23$ & $1.07 \pm 0.33$ & 1.024 & 0.752 \\
\hline
\end{tabular}

$\mathrm{GH}=$ growth hormone; $\mathrm{PRL}=$ prolactin; $\mathrm{LH}=$ luteinizing hormone; $\mathrm{FSH}=$ follicle-stimulating hormone; $\mathrm{TSH}=$ thyroidstimulating hormone.

\section{DISCUSSION}

The pituitary, a major component of the neuroendocrine system, communicates between the hypothalamus and peripheral target organs, and plays an important role in ensuring normal growth and metabolism in the body. As it involves complex processes, the human body development can be severely compromised by abnormal development of the pituitary (Dateki et al., 2010; Qi Wei et al., 2011; Skarnes et al., 2011). In this study, the pituitary development-related transcriptional factor ZBTB20 was shown to express from E14 stage until birth. This was abundantly distributed in both the anterior and posterior regions of the pituitary even in adult mice. At E15 or E16, the number of ZBTB20-positive cells increased significantly. As this is also the differentiation time of $\mathrm{GH}$ and $\mathrm{PRL}$, it can be deduced that ZBTB20 expression occurred before the differentiation of these two hormones, suggesting the potential participation of ZBTB20 in this process, and in maintaining normal morphology and function of pituitary (El Chehadeh et al., 2010; Di lorgi et al., 2012b; Harrington and Palmert, 2012). 
The development of the pituitary gland persists from the embryonic stage and postnatal differentiation and proliferation of endocrine cells. The embryonic pituitary development is under the direction of multiple signaling molecules and transcriptional factors. Previous studies have indicated that major regulatory factors are located in the hypothalamus and craniopharyngeal pouch. Postnatal pituitary development, however, is largely dependent on feedback from both hypothalamus and peripheral target glands, both of which exert similar regulatory effects on endocrinal cells at various stages (Smyczynska et al., 2010; Kalina et al., 2012; Zacharin et al., 2012). In this study, a ZBTB20 gene KO mouse model was generated, in which the developmental and functional features of the pituitary were observed. Both real-time PCR and western blot assays showed barely any PRL mRNA or protein in the pituitary of KO mice, and only half the normal levels of $\mathrm{GH}$. The decrease in $\mathrm{GH}$ in adult $\mathrm{KO}$ mice (>3 weeks old) may be due to the enhanced negative or suppressed positive feedback signals from the pituitary, rather than the endogenous $\mathrm{KO}$ of the ZBTB20 gene (Nevalainen et al., 2004).

This study revealed the importance of PRL- and GH-releasing cells in the development of the pituitary; however, the fate of genetic factors regulating differentiation and the detailed mechanism remain largely unclear. Currently, the possibility of reciprocal transformation between GH and PRL after birth remains to be validated. Previous studies have shown that GH and PRL cells may be inter-changeable, as progenitors may differentiate into $\mathrm{GH}$, which is later transformed into PRL (Di lorgi et al., 2012a; Tsai et al., 2012). Recent studies in fish and birds have supported the theory that PRL and GH follow an independent differentiation (Jagtap et al., 2012; Takagi et al., 2012). Other studies, however, did not detect the expression of PRL in the embryonic pituitary (Garcia Martin et al., 2010; Sukhija et al., 2012). Our study confirmed the existence of PRL at E15 and E16 in mouse adenohypophysis. Such PRL-releasing cells consisted of both PRL- and GH-positive cells, suggesting both environmental and cellular effects on the final differentiation route of such cells.

In summary, this study confirmed the importance of ZBTB20 in regulating pituitary development. The gene KO of ZBTB20 can result in under-development of adenohypophysis and abnormal differentiation of GH and PRL. Future treatment strategies could be made to target this transcriptional factor. The results of this study would facilitate better understanding of the role and regulatory mechanism of ZBTB20 in adenohypophysis.

\section{Conflicts of interest}

The authors declare no conflict of interest.

\section{REFERENCES}

Baranowski E and Hogler W (2012). An unusual presentation of acquired hypothyroidism: the Van Wyk-Grumbach syndrome. Eur. J. Endocrinol. 166: 537-542.

Cabrera SM, DiMeglio LA and Eugster EA (2013). Incidence and characteristics of pseudoprecocious puberty because of severe primary hypothyroidism. J. Pediatr. 162: 637-639.

Dateki S, Kosaka K, Hasegawa K, Tanaka H, et al. (2010). Heterozygous orthodenticle homeobox 2 mutations are associated with variable pituitary phenotype. J. Clin. Endocrinol. Metab. 95: 756-764.

Di lorgi N, Allegri AE, Napoli F, Bertelli E, et al. (2012a). The use of neuroimaging for assessing disorders of pituitary development. Clin. Endocrinol. 76: 161-176.

Di lorgi N, Napoli F, Allegri AE, Olivieri I, et al. (2012b). Diabetes insipidus - diagnosis and management. Horm. Res. Paediatr. 77: 69-84.

El Chehadeh S, Bensignor C, de Monleon JV, Mejean M, et al. (2010). The pituitary stalk interruption syndrome: endocrine features and benefits of growth hormone therapy. Ann. Endocrinol. 71: 102-110. 
Franceschi R, Rozzanigo U, Failo R, Bellizzi M, et al. (2011). Pituitary hyperplasia secondary to acquired hypothyroidism: case report. Ital. J. Pediatr. 37: 15.

Garcia Martin A, Cortes Berdonces M, Tenorio Jimenez C and Torres Vela E (2010). Perinatal adverse events and neuroanatomical abnormalities in patients with idiopathic hypopituitarism. Endocrinol. Nutr. 57: 251-255.

Harrington J and Palmert MR (2012). Clinical review: Distinguishing constitutional delay of growth and puberty from isolated hypogonadotropic hypogonadism: critical appraisal of available diagnostic tests. J. Clin. Endocrinol. Metab. 97: 3056-3067.

Jagtap VS, Acharya SV, Sarathi V, Lila AR, et al. (2012). Ectopic posterior pituitary and stalk abnormality predicts severity and coexisting hormone deficiencies in patients with congenital growth hormone deficiency. Pituitary 15: 243-250.

Kalina MA, Kalina-Faska B, Gruszczynska K, Baron J, et al. (2012). Usefulness of magnetic resonance findings of the hypothalamic-pituitary region in the management of short children with growth hormone deficiency: evidence from a longitudinal study. Childs. Nerv. Syst. 28: 121-127.

Nanbu-Wakao R, Fujitani Y, Masuho Y, Muramato M, et al. (2000). Prolactin enhances CCAAT enhancer-binding protein-beta (C/EBP beta) and peroxisome proliferator-activated receptor gamma (PPAR gamma) messenger RNA expression and stimulates adipogenic conversion of NIH-3T3 cells. Mol. Endocrinol. 14: 307-316.

Nevalainen MT, Xie J, Torhorst J, Bubendorf L, et al. (2004). Signal transducer and activator of transcription-5 activation and breast cancer prognosis. J. Clin. Oncol. 22: 2053-2060.

Qi Wei P, Hui Juan Z, Feng Ying G, Na Shi L, et al. (2011). Magnetic resonance image of sellar region in pituitary stalk interruption syndrome in children and adolescents. Zhongguo Yi. Xue. Ke. Xue. Yuan. Xue. Bao. 33: 9-13.

Reynaud R, Albarel F, Saveanu A, Kaffel N, et al. (2011). Pituitary stalk interruption syndrome in 83 patients: novel HESX1 mutation and severe hormonal prognosis in malformative forms. Eur. J. Endocrinol. 164: 457-465.

Shillingford JM, Miyoshi K, Robinson GW, Bierie B, et al. (2003). Proteotyping of mammary tissue from transgenic and gene knockout mice with immunohistochemical markers: a tool to define developmental lesions. J. Histochem. Cytochem. 51: 555-565.

Skarnes WC, Rosen B, West AP, Koutsourakis M, et al. (2011). A conditional knockout resource for the genome-wide study of mouse gene function. Nature 474: 337-342.

Smyczynska J, Hilczer M, Stawerska R, Lewinsky A, et al. (2010). Thyroid function in children with growth hormone (GH) deficiency during the initial phase of $\mathrm{GH}$ replacement therapy - clinical implications. Thyroid Res. 3: 2.

Sukhija K, Pyne M, Ali S, Orr V, et al. (2012). Developing an extended genomic engineering approach based on recombineering to knock-in heterologous genes to Escherichia coli genome. Mol. Biotechnol. 51: 109-118.

Takagi M, Ishii T, Inokuchi M, Amano N, et al. (2012). Gradual loss of ACTH due to a novel mutation in LHX4: comprehensive mutation screening in Japanese patients with congenital hypopituitarism. PLoS One 7: e46008.

Tsai SL, Laffan E and Lawrence S (2012). A retrospective review of pituitary MRI findings in children on growth hormone therapy. Pediatr. Radiol. 42: 799-804.

Wu W, Coss D, Lorenson MY, Kuo CB, et al. (2003). Different biological effects of unmodified prolactin and a molecular mimic of phosphorylated prolactin involve different signaling pathways. Biochemistry 42: 7561-7570.

Zacharin M, Sabin MA, Nair VV and Dabadghao P (2012). Addition of recombinant follicle-stimulating hormone to human chorionic gonadotropin treatment in adolescents and young adults with hypogonadotropic hypogonadism promotes normal testicular growth and may promote early spermatogenesis. Fertil. Steril. 98: 836-842. 